proliferation ( $\mathrm{p}=0.0043$ ) compared with LRIG1-negative cells. Similarly, LRIG1-expressing human airway basal cells isolated from endobronchial brush biopsy samples exhibit increased colony-forming capacity $(\mathrm{p}=0.0469)$. Topical application of NTCU to mice recapitulates the development of human pre-invasive and SCLC lesions after 23 weeks. Results show lesions in LRIG1-KO mice to be larger than those of WT animals. Knock down of LRIG1 in cultured human airway basal cells alters cell phenotype, leading to an increased colony-forming efficiency and greater proliferation at cell confluence.

Conclusions LRIG1 has an important role in stem cell homeostasis of the human and murine airway epithelium. Loss of LRIG1 promotes pre-cancerous lesion development in a murine SqCLC mouse model and behaviour of human epithelial cells in culture, indicating a potential target for chemoprevention of SqCLC in humans.

\section{S10 INVESTIGATION OF VESSEL STRUCTURE IN THE VICINITY OF LUNG TUMOURS}

N Sadri, D Wertheim. Kingston University, Kingston, Surrey, UK

10.1136/thoraxjnl-2016-209333.16

Lung cancer is considered a major cause of cancer death. ${ }^{1}$ We are currently developing methods for detection of vessels in lung CT images. The aim of this study was to investigate the number of vessels in areas of unilateral lung tumours and compare with the equivalent contralateral lung with no tumour. Lung CT images were downloaded from the Cancer Imaging Archive wiki.cancerimagingarchive.net/display/Public/LungCT-Diagnosis. ${ }^{2} \quad$ Software was written in MATLAB (The MathWorks Inc., USA) in order to display and analyse the DICOM images. Windowing was performed manually in order to clearly display the tumours as well as surrounding vessel like structures. Using the software eight sets of images were analysed; the number of clearly defined vessel like structures directly attached to the tumour were counted and compared with the corresponding region in the contralateral lung with no evidence of tumour; small vessel like structure and branches were not included. The area of the tumour was manually delineated and calculated in terms of pixels. For each set of CT images, one image was used where the tumour size was greatest. In all eight cases the number of clear vessel like structures in the immediate vicinity of the tumour was greater than that in the corresponding area on the contralateral side, mean (standard deviation) of the difference 5 (1.6), there was a significant difference $\mathrm{p}<0.001$ (one sample $\mathrm{t}$ test). In addition vessel like structures often appeared brighter on the side of the tumour. The results of this pilot study suggest that the number of clear bright vessel like structures in the immediate vicinity of a lung tumour may be higher than in the corresponding area on the contralateral side. We feel this research merits further study in order to investigate if this approach may help enable early detection of lung tumours.

\section{REFERENCES}

1 Siegel $R$, et al. Cancer statistics, 2013. CA Cancer J Clin 2013:63:11-30.

2 Grove 0, et al. Data from: Quantitative computed tomographic descriptors associate tumour shape complexity and intratumor heterogeneity with prognosis in lung adenocarcinoma. The Cancer Imaging Archive (2015). http://dx.doi.org/10.7937/ K9/TCIA.2015.A6V7JIWX

\section{Progress in the ITU}

\section{S11 DECREASED ANTI-INFLAMMATORY POTENTIAL OF MESENCHYMAL STEM CELLS AFTER PROLONGED IN VITRO EXPANSION WILL IMPACT ON THEIR USE AS A THERAPY FOR ACUTE RESPIRATORY DISTRESS SYNDROME}

M Aslani, RY Mahida, A Scott, DR Thickett. University of Birmingham, Birmingham, UK

\subsection{6/thoraxjnl-2016-209333.17}

Introduction and objectives Mesenchymal stem cells (MSC) have well-established anti-inflammatory properties and could potentially be used therapeutically in Acute Respiratory Distress Syndrome (ARDS). ${ }^{1}$ MSC conditioned medium (MSC-CM) has been found to reproduce the beneficial effects of MSCs. ${ }^{2}$ However, the impact of expansion on MSC secretome remains elusive. In the present study we assessed the expression of four potent MSC paracrine factors after prolonged in vitro culture, while investigating the effects of passaging on the in vitro properties of MSCs.

Methods Human Bone marrow-derived MSCs were expanded in vitro in $\alpha \mathrm{MEM}$ with $16 \%$ FBS. Conditioned medium was collected from each passage and stored at $-40^{\circ} \mathrm{C}$. Scratch assays were undertaken using A549 cells treated with MSC-CM and control media. Proliferation of A549 cells was assessed via BrdU assay. Gene expression of expanded MSCs was assessed by RTqPCR. VEGF and Angiopoietin in the MSC-CM were quantified by ELISA.

Results RT-qPCR revealed that MSCs express the anti-inflammatory genes PTGES2, FGF7 and ANGPT1. The expression of these genes doubled after 8 days in culture and subsequently decreased. $(\mathrm{P}<0.0005$, one way Anova, $\mathrm{n}=2)$. The secretion of Angiopoietin and VEGF decreased with prolonged expansion (Figure 1). MSC-CM obtained after 8 days of culture induced more efficient wound healing compared to MSC-CM obtained following prolonged expansion $(\mathrm{P}<0.005$, one way Anova, $\mathrm{n}=2$ ). However, MSC-CM failed to affect the proliferation of A549 cells.

Conclusion We conclude that MSCs of early passages are more potent angiogenic inducers, while promoting wound healing in

\section{VEGF secretion}

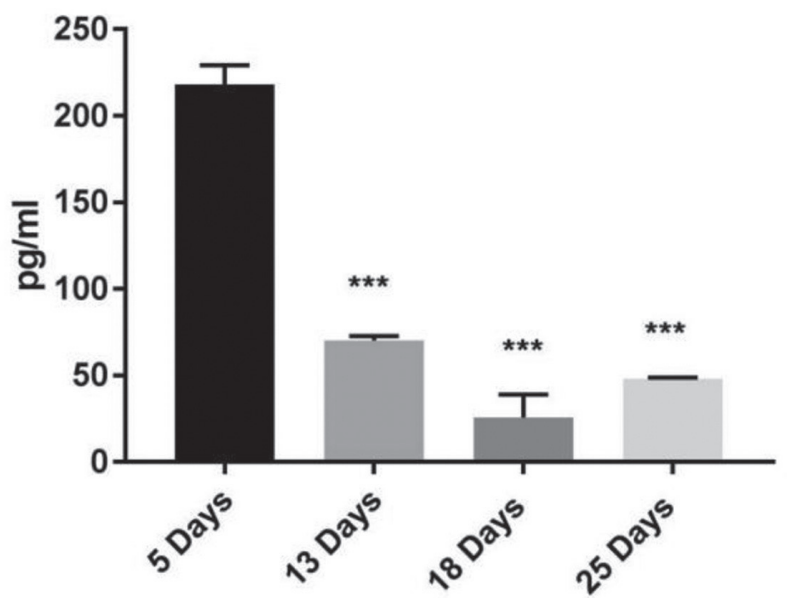

Abstract S11 Figure 1 Levels of secreted VEGF decrease after prolonged in vitro culture $\left({ }^{*} \mathrm{P}<0.05,{ }^{* *} \mathrm{P}<0.0005, \mathrm{n}=2\right)$ 
vitro. Thus, we provide important insights into the changes occurring after prolonged in vitro expansion, underlining the importance of using low passage MSCs in clinical trials for ARDS. In agreement with published data, we also found that MSCs do not induce cellular proliferation in the absence of stimulation.

\section{REFERENCES}

1 Antunes MA, et al. Mesenchymal stem cell trials for pulmonary diseases. I Cell Biochem 2014;115:1023-1032.

2 Kordelas L, et al. MSC-derived exosomes: a novel tool to treat therapy-refractory graft-versus-host disease. Leukaemialeukemia 2014;28:970-973.

\section{S12 PLASMA SYNDECAN-1 LEVEL AS A PREDICTIVE MARKER OF VASOPLEGIA ASSOCIATED WITH SURGERY REQUIRING CARDIOPULMONARY BYPASS AND POSSIBLE INVOLVEMENT OF OXIDATIVE STRESS}

${ }^{1} \mathrm{MG}$ Rasiah, ${ }^{1} \mathrm{C}$ Michaeloudes, ${ }^{1} \mathrm{~T}$ Svermova, ${ }^{1} \mathrm{Z}$ Nikolakopoulou, ${ }^{2} \mathrm{~B}$ Creagh-Brown, ${ }^{1}$ PK Bhavsar, 'A Burke-Gaffney. 'Imperial College London, London, UK; ${ }^{2}$ The Royal Surrey County Hospital NHS Foundation Trust, Guildford, UK

\subsection{6/thoraxjnl-2016-209333.18}

Background Vasoplegic syndrome (severe refractory hypotension) is associated with oxidative stress leading to endothelial dysfunction and complicates 10 to $40 \%$ of surgery requiring cardiopulmonary bypass (CPB). Whilst operative mortality is low, recovery is often prolonged in patients developing vasoplegia. There are, as yet, no validated biomarkers for vasoplegia that could be used to identify 'at risk' patients. We hypothesised that plasma levels of the endothelial surface layer (glycocalyx) protein, syndecan-1, shed during $\mathrm{CPB}$, will be higher in patients who develop vasoplegia and that leukocyte responses to oxidative stress will be altered.

Methods Patients $(n=48)$ undergoing cardiac surgery requiring $\mathrm{CPB}$ were, prospectively, enrolled; blood collected and indices of outcome recorded. A surrogate index of vasoplegia was adopted: requirement for infusion of vasoconstrictor agents for longer than 48h. An enzyme-linked immunosorbent assay was used to measure plasma levels of syndecan-1 at four time-points: after induction of anaesthesia but before CPB (T1); within $30 \mathrm{~min}$ of
$\mathrm{CPB}$ ending (T2); $2 \mathrm{~h}$ (T3) and 24h (T4) post-CPB. Real time qPCR was used to determine, in patient leukocytes $(n=20)$, relative expression (to house-keeping gene18S) of mRNA for markers of oxidative stress; NQO1 and SOD2, cytoplasmic and mitochondrial enzymes, respectively; and for comparison, TNF $\alpha$. Results Syndecan-1 levels at T2 were significantly higher in vasoplegic patients $(110.7 \mathrm{ng} / \mathrm{mL}$, IQR 65.46-155.2) than non-vasoplegic patients $(53.8 \mathrm{ng} / \mathrm{mL}$, IQR 40.67-102.2; $<<0.001)$. ROC curve analysis showed syndecan-1 had significant $(\mathrm{p}=0.009)$ predictive power for onset of vasoplegia, with an area under the curve of 0.766 (95\% CI: 0.6019-0.9301); and a cut-off of 63.33 $\mathrm{ng} / \mathrm{mL}$ (83.33\% sensitivity, 69.23\% specificity). Syndecan-1 levels were higher in patients whose intensive care unit length of stay (LOS) and hospital LOS were above corresponding medians for the cohort ( $\mathrm{p}=0.0061$ and $\mathrm{p}=0.0148$, respectively). NQO1 relative expression was significantly higher $(\mathrm{p}=0.022)$ in vasoplegic patients $(3.779 \pm 1.036)$ than non-vasoplegic patients (1.3 \pm 0.302); whereas, neither SOD2 nor TNF $\alpha$ expression were significantly altered.

Conclusion Plasma syndecan-1 measured immediately post-CPB had good predictive power for patients at risk of vasoplegia. Greater relative expression of leukocyte NQO1 in vasoplegic patients indicates activation of antioxidant defence mechanisms in response to oxidative stress, which could contribute to syndecan-1 shedding.

\section{S13 PHARMACOKINETICS AND PHARMACODYNAMICS OF ANTIMICROBIALS IN CRITICALLY ILL PATIENTS WITH LOWER RESPIRATORY TRACT INFECTIONS. ARE 'ONE SIZE FITS ALL' DOSES APPROPRIATE?}

${ }^{1}$ IB Oldfield, ${ }^{1} \mathrm{~K}$ Kipper, ${ }^{1} \mathrm{Cl}$ Barker, ${ }^{1} \mathrm{BJ}$ Philips, ${ }^{2} \mathrm{M}$ Cecconi, ${ }^{2} \mathrm{~A}$ Rhodes, ${ }^{1} \mathrm{~A}$ Johnston, ${ }^{3} \mathrm{JF}$ Standing, ${ }^{1} \mathrm{EH}$ Baker, ${ }^{1} \mathrm{M}$ Sharland, ${ }^{1} \mathrm{DO}$ Lonsdale. ${ }^{1}$ Institute for Infection and Immunity, St George's, University of London, London, UK; ${ }^{2}$ St George's University Hospitals NHS Foundation Trust, London, UK; ${ }^{3}$ Infectious Diseases and Microbiology Unit, University College London, Institute of Child Health, London, UK

\subsection{6/thoraxjnl-2016-209333.19}

Introduction Respiratory infection is a common cause of severe sepsis. ${ }^{1}$ Current therapeutic guidelines emphasise the importance of early initiation of antibiotic therapy, but make no
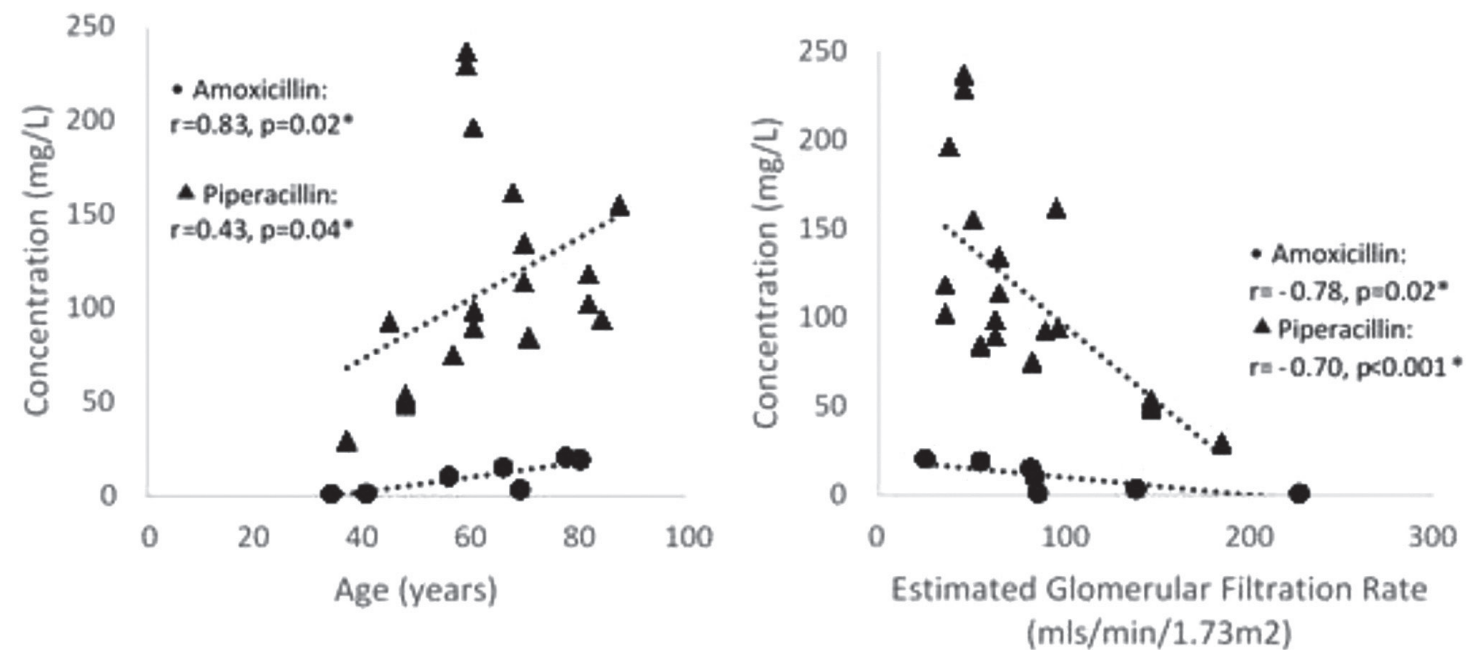

Abstract S13 Figure 1 Antimicrobial concentration measured at 50\% of the dosing interval plotted against age (left) and eGFR (right). Line of best fir and associated coefficients suggest correlation with age and negative correlation with eGFR 